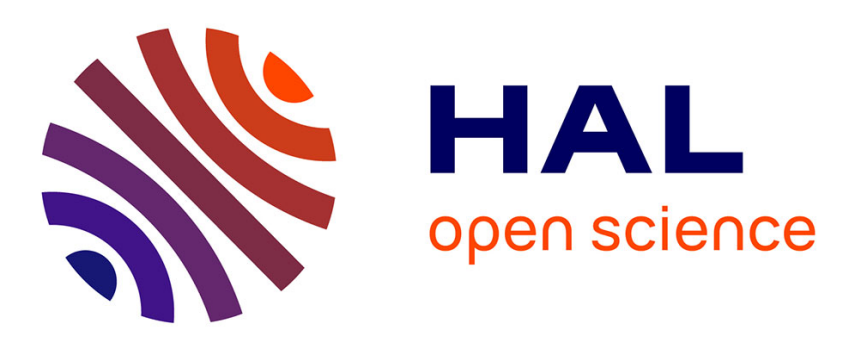

\title{
Closed loop output error identification with bounded disturbances
}

Mathieu Pouliquen, Olivier Gehan, Eric Pigeon, Miloud Frikel

\section{To cite this version:}

Mathieu Pouliquen, Olivier Gehan, Eric Pigeon, Miloud Frikel. Closed loop output error identification with bounded disturbances. IFAC Symposium on System Identification 2012, Jul 2012, Brussels, Belgium. pp.870-875. hal-00771453

\section{HAL Id: hal-00771453 https://hal.science/hal-00771453}

Submitted on 8 Jan 2013

HAL is a multi-disciplinary open access archive for the deposit and dissemination of scientific research documents, whether they are published or not. The documents may come from teaching and research institutions in France or abroad, or from public or private research centers.
L'archive ouverte pluridisciplinaire HAL, est destinée au dépôt et à la diffusion de documents scientifiques de niveau recherche, publiés ou non, émanant des établissements d'enseignement et de recherche français ou étrangers, des laboratoires publics ou privés. 


\title{
Closed loop output error identification with bounded disturbances.
}

\author{
Mathieu Pouliquen, Olivier Gehan, Eric Pigeon, \\ Miloud Frikel \\ Control Group, GREYC CNRS UMR 6072 \\ ENSICAEN, 06 Bd du Marechal Juin \\ 14050 Caen Cedex, France \\ mathieu.pouliquen@unicaen.fr
}

\begin{abstract}
The problem of closed loop system identification in presence of bounded disturbances is considered. In this paper two recursive algorithms are proposed to solve this identification problem. Stability and convergence properties are demonstrated. Simulation results validate the proposed solutions.
\end{abstract}

\section{INTRODUCTION}

In recent decades the identification of closed loop systems has received much interest as shown by the following references: [18], [20] [13], [6], [3], [9]. The interest is motivated because for many industrial processes open loop experiments are prohibited (safety, stability, efficiency of operation, etc.) but also for reasons of supervision (the desired performance are met?). All practical identification algorithms have to deal with measurements corrupted by noise and to the best of our knowledge, most of the works in the closed loop identification literature consider the stochastic noise assumption. In some cases (unknown probability distribution of the disturbances, modeling inaccuracy) such assumption cannot be done and the bounded noise assumption seems to be more appropriate.

To address this identification problem we will take inspiration from the identification method CLOE (Closed Loop Output Error) presented in [10], [11] and [12]. This method will be adapted by considering the Set Membership Identification (SMI) algorithms (see [15], [14], [21], [7], [8] and [2]) and particularly the Optimal Bounding Ellipsoid (OBE) algorithms. The computational complexity of these algorithms is low and they are appropriated to handle the identification problem in presence of bounded disturbances. Their principle consists in the estimation of a feasible set of parameters which must be consistent with the measurement data and the model structure. Important contributions have been presented in [5], [4], [1] and [19]. The paper will focus on the extension of some stability and convergence results ([1], [19], [16]) to closed loop output error model.

The paper is organized as follows: in section II the identification problem is posed. In section III, recursive estimation algorithms are derived and and their convergence analysis are addressed. Some simulation results are given in section IV. Finally, section V concludes the paper.

\section{PROBLEM FORMULATION AND NOTATION}

Consider the closed loop depicted in Fig. 1 where the plant is a linear system which can be described by the transfer function $G_{o}(q) . r_{t}, u_{t}$ and $y_{t}$ are respectively an exogenous input, the system input and the system output. $w_{t}$ denotes the output disturbances and $C(q)$ a linear controller. The underlying output behavior is given by:

$$
y_{t}=\frac{G_{o}(q)}{1+G_{o}(q) C(q)} r_{t}+\frac{1}{1+G_{o}(q) C(q)} w_{t}
$$

Here, it is assumed that there exists a discret time transfer function $G(q)$ stabilizing the closed loop and such that the output $y_{t}$ is given by

$$
y_{t}=\frac{G(q)}{1+G(q) C(q)} r_{t}+v_{t}
$$

where $v_{t}$ is an unknown and bounded disturbing term (noise measurement, state disturbances, modeling inaccuracy, etc.):

$$
v_{t}=\left(\frac{G_{o}(q)}{1+G_{o}(q) C(q)}-\frac{G(q)}{1+G(q) C(q)}\right) r_{t}+\frac{1}{1+G_{o}(q) C(q)} w_{t}
$$

We assume that an upper bound is supposed to be available on $v_{t}$ :

$$
\left|v_{t}\right| \leq \delta_{v_{t}}
$$

The model $G(q)$ is defined by

$$
G(q)=q^{-d} \frac{B(q)}{A(q)}
$$

with

$$
\left\{\begin{array}{l}
B(q)=b_{0}+b_{1} q^{-1}+\cdots+b_{n_{b}} q^{-n_{b}} \\
A(q)=1+a_{1} q^{-1}+\cdots+a_{n_{a}} q^{-n_{a}}
\end{array}\right.
$$

and the identification problem treated in this paper is stated as: estimate the parameters $\left\{b_{i}\right\}$ and $\left\{a_{i}\right\}$ of the model by using the available data $\left\{r_{t}, y_{t}\right\}$, the knowledge of the controller $C(q)$ and the upper bound $\delta_{v_{t}}$.

The presented identification algorithms are similar to the CLOE algorithm described in [10], [11] and [12]. Here, the difference lies in the nature of the noise component: the noise is not modeled as a stochastic process, the only information is the known upper bound $\delta_{v_{t}}$. This will have a strong impact on the stability analysis. 


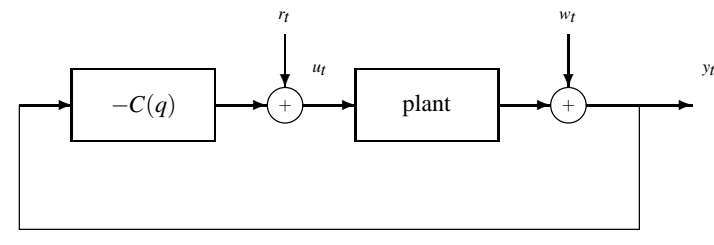

Fig. 1. Closed loop system

The one-step-ahead output predictor is defined bearing in mind the optimal predictor structure (see [13]), namely

$$
\hat{y}_{t}=y_{t}-v_{t}=\phi_{t}^{T} \theta^{*}
$$

with

$$
\theta^{* T}=\left(\begin{array}{llllll}
a_{1} & \cdots & a_{n_{a}} & b_{0} & \cdots & b_{n_{b}}
\end{array}\right)
$$

$\theta^{*} \in \mathbb{R}^{n}$ is the unknown parameters vector to be identified with $n=n_{a}+n_{b}+1$ the number of parameters.

$\phi_{t}$ is defined by

$$
\phi_{t}^{T}=\left(\begin{array}{cccccc}
-\hat{y}_{t-1} & \cdots & -\hat{y}_{t-n_{a}} & \hat{u}_{t-d} & \cdots & \hat{u}_{t-d-n_{b}}
\end{array}\right)
$$

where

$$
\hat{u}_{t}=r_{t}-C(q) \hat{y}_{t}
$$

and

$$
C(q)=\frac{R(q)}{S(q)}
$$

A key observation is that the output predictor $\hat{y}_{t}$ is not linear in the system parameters, this will determine the stability of the identification algorithm.

\section{IDENTIFICATION ALGORITHMS}

\section{A. The CLOE-OBE (Closed Loop Output Error - OBE)} algorithm.

This paper focuses on the design of a recursive identification algorithm for the system described by (2), (3) and (4). Before introducing the parameter adaptation algorithm, let us define the well known a priori and a posteriori predictors as

$$
\left\{\begin{array}{l}
\hat{y}_{t / t-1}=\hat{\phi}_{t}^{T} \hat{\theta}_{t-1} \\
\hat{y}_{t / t}=\hat{\phi}_{t}^{T} \hat{\theta}_{t}
\end{array}\right.
$$

$\hat{\theta}_{t}$ represents the estimation of the parameters vector at the actual time $t$ while $\hat{\phi}_{t}$ is an estimate of the prediction data vector $\phi_{t}$ which is simply obtained by replacing the unknown components $\hat{y}_{t-i}$ by their a posteriori estimates $\hat{y}_{t-i / t-i}$ and $\hat{u}_{t-d-i}$ by their a posteriori estimates $\hat{u}_{t-d-i / t-d-i}$ :

$\hat{\phi}_{t}^{T}=\left(\begin{array}{llllll}-\hat{y}_{t-1 / t-1} & \cdots & -\hat{y}_{t-n_{a} / t-n_{a}} & \hat{u}_{t-d / t-d} & \cdots & \hat{u}_{t-d-n_{b} / t-d-n_{b}}\end{array}\right)$

with

$$
\hat{u}_{t-i / t-i}=r_{t-i}-C(q) \hat{y}_{t-i / t-i}
$$

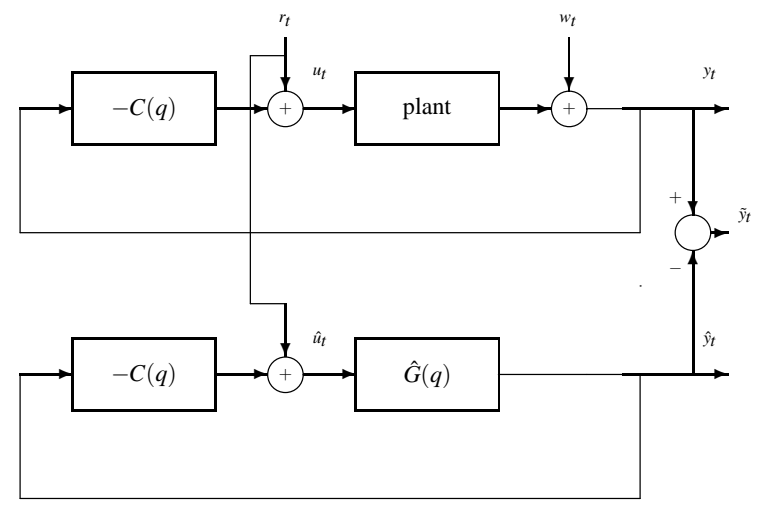

Fig. 2. Closed loop identification scheme

From these adjustable predictors we get the following closed loop a priori and a posteriori prediction errors:

$$
\left\{\begin{array}{l}
\varepsilon_{t / t}=y_{t}-\hat{y}_{t / t-1} \\
\varepsilon_{t / t-1}=y_{t}-\hat{y}_{t / t}
\end{array}\right.
$$

A key observation is that the a posteriori prediction error $\varepsilon_{t / t}$ can be expressed as:

$$
\begin{gathered}
\varepsilon_{t / t}=y_{t}-\hat{\phi}_{t}^{T} \hat{\theta}_{t} \\
\varepsilon_{t / t}=\phi_{t}^{T} \theta^{*}+v_{t}-\hat{\phi}_{t}^{T} \hat{\theta}_{t}+\left(\hat{\phi}_{t}^{T} \theta^{*}-\hat{\phi}_{t}^{T} \theta^{*}\right)
\end{gathered}
$$

Bearing in mind structures of $\phi_{t}$ and $\hat{\phi}_{t}$ this gives

$$
\varepsilon_{t / t}=\frac{S(q)}{A(q) S(q)+q^{-d} B(q) R(q)} \hat{\phi}_{t}^{T} \tilde{\theta}_{t}+v_{t}
$$

with $\tilde{\theta}_{t}=\theta^{*}-\hat{\theta}_{t}$.

It will be seen later that this relationship is fundamental for establishing the stability and convergence properties of the identification algorithm.

The estimated parameters vector $\hat{\theta}_{t}$ has to maintain the closed loop output error below a bound defined from the upper bound on the disturbance $v_{t}$. Under some conditions presented below, the following Closed Loop Output Error OBE algorithm provides such estimation:

$$
\left\{\begin{array}{l}
\hat{\theta}_{t}=\hat{\theta}_{t-1}+\Gamma_{t} \varepsilon_{t / t-1} \\
\Gamma_{t}=\frac{P_{t-1} \hat{\phi}_{t} \sigma_{t}}{\lambda+\hat{\phi}_{t}^{T} P_{t-1} \hat{\phi}_{t} \sigma_{t}} \\
P_{t}=\frac{1}{\lambda}\left(I_{n}-\Gamma_{t} \hat{\phi}_{t}^{T}\right) P_{t-1} \\
\varepsilon_{t / t-1}=y_{t}-\hat{\phi}_{t}^{T} \hat{\theta}_{t-1}
\end{array}\right.
$$

where $0<\lambda \leq 1$ is a design parameter forgetting factor that will be used to monitor the parameter adaptation dynamics. $\sigma_{t}$ is a switching flag given by:

$$
\sigma_{t}=\left\{\begin{array}{l}
\frac{\lambda}{\hat{\phi}_{t}^{T} P_{t-1} \hat{\phi}_{t}}\left(\left|\frac{\varepsilon_{t / t-1}}{\delta_{t}}\right|-1\right) \\
\text { if }\left(\left|\varepsilon_{t / t-1}\right|>\delta_{t}\right) \text { and }\left(\hat{\phi}_{t}^{T} P_{t-1} \hat{\phi}_{t}>0\right) \\
0 \text { otherwise }
\end{array}\right.
$$


$\delta_{t}$ is a user defined positive scalar.

From (6) the a posteriori prediction error $\varepsilon_{t / t}$ can be written as

$$
\varepsilon_{t / t}=\left(1-\hat{\phi}_{t}^{T} \Gamma_{t}\right) \varepsilon_{t / t-1}
$$

which can also be rewritten, by using the expression for $\Gamma_{t}$, as:

$$
\varepsilon_{t / t}=\frac{\lambda}{\lambda+\hat{\phi}_{t}^{T} P_{t-1} \hat{\phi}_{t} \sigma_{t}} \varepsilon_{t / t-1}
$$

Using the value of $\sigma_{t}$ for $\sigma_{t} \neq 0$ (8) yields:

$$
\left|\varepsilon_{t / t}\right|=\delta_{t}
$$

Provided that the persistent excitation condition is satisfied (i.e. $\hat{\phi}_{t}^{T} P_{t-1} \hat{\phi}_{t}>0$ ), this clearly shows that the CLOE-OBE algorithm ensures the following key property:

$$
\left\{\begin{array}{l}
\text { if }\left|\varepsilon_{t / t-1}\right|>\delta_{t} \text { then } \sigma_{t} \neq 0 \text { and }\left|\varepsilon_{t / t}\right|=\delta_{t} \\
\text { if }\left|\varepsilon_{t / t}\right| \leq \delta_{t} \text { then } \sigma_{t}=0 \text { and }\left|\varepsilon_{t / t}\right| \leq \delta_{t}
\end{array}\right.
$$

$\delta_{t}$ is then a bound on the a posteriori adaptation error which has to be specified taking into account the bound $\delta_{v_{t}}$.

The results of the stability analysis are presented in the following theorem. These results are similar to the stability analysis of the Output Error - OBE algorithm used for plant model identification in open loop and presented in [16].

Result 1: Consider the class of systems defined in section II and the CLOE-OBE algorithm given by (6) and (7). Assume that

- $G(q)$ is such that

$$
\left\|1-\frac{A(q) S(q)+q^{-d} B(q) R(q)}{S(q)}\right\|_{1}<1
$$

where $\|\cdot\|_{1}$ is the $l_{1}$ induced norm;

- $\delta_{t}$ is such that:

$$
\delta_{t} \geq \frac{\left\|\frac{A(q) S(q)+q^{-d} B(q) R(q)}{S(q)}\right\|_{1}}{1-\left\|1-\frac{A(q) S(q)+q^{-d} B(q) R(q)}{S(q)}\right\|_{1}} \delta_{v_{t}}
$$

then for all initial conditions

$$
\left|\tilde{\theta}_{t}\right|^{2} \leq \gamma_{1}\left|\tilde{\theta}_{0}\right|^{2}
$$

with $\gamma_{1}=\frac{\lambda_{\max }\left(P_{0}^{-1}\right)}{\lambda_{\min }\left(P_{0}^{-1}\right)}$.

where $\lambda_{\max }\left(P_{0}^{-1}\right)$ and $\lambda_{\min }\left(P_{0}^{-1}\right)$ are respectively the maximum and the minimum eigenvalues of $P_{0}^{-1}$.

If, furthermore, $\left\{\hat{\phi}_{t}\right\}$ is a persistently exciting sequence of order $o_{e} \geq n$, i.e there exist $\alpha>0$ and $\beta>0$ such that for all $t$

$$
\alpha I_{n} \leq \sum_{i=0}^{o_{e}-1} \hat{\phi}_{t+i} \sigma_{t+i} \hat{\phi}_{t+i}^{T} \leq \beta I_{n}
$$

Then the following properties hold:
- for all $t \geq o_{e}+1$

$$
\left|\tilde{\theta}_{t}\right|^{2} \leq \gamma_{2} \lambda^{t}\left|\tilde{\theta}_{0}\right|^{2}
$$

with

$$
\gamma_{2}= \begin{cases}\frac{\lambda_{\max }\left(P_{0}^{-1}\right)}{\alpha}\left(\frac{\lambda^{-o_{e}}-1}{\lambda^{-1}-1}\right) & \text { if } \lambda<1 \\ \frac{\lambda_{\max }\left(P_{0}^{-1}\right)}{\alpha} & \text { if } \lambda=1\end{cases}
$$

- For $\lambda<1$ one has

$$
\lim _{t \rightarrow \infty}\left|\varepsilon_{t / t-1}\right| \leq \delta_{t}
$$

Proof: For lack of space we do not detail the proof. The form of the adaptation algorithm and the relation (5) between $\varepsilon_{t / t}$ and $\tilde{\theta}_{t}$ allows to use results established in [16].

In a first part of the proof, it is shown that the Lyapounov function $V_{t}=\tilde{\theta}_{t}^{T} P_{t}^{-1} \tilde{\theta}_{t}$ is such that $V_{t} \leq \lambda^{t} V_{0}$ if conditions (9) and (10) are satisfied.

In a second part property (11) is obtained using the fact that $P_{t}^{-1} \geq \lambda^{t} P_{0}^{-1}$. Finally, the persistent excitation condition is used to get an other bound on $P_{t}^{-1}$, this gives properties (13) and (14).

Remark 1: The conditions (9) and (10) are only sufficient conditions and we have observed the algorithm to work well in some cases where these conditions are not satisfied.

Remark 2: The condition (9) implies the asymptotic stability of the controller used during the identification step.

Remark 3: The ability to reach the true parameters vector depends on the threshold $\delta_{t}$, thus its specification proves to be particularly crucial. The design of this threshold is influenced by the system throughout the polynomials $A(q)$ and $B(q)$ (which are unknown objects), by the controller and by the disturbances effects throughout the bound $\delta_{v_{t}}$. A dichotomy-based procedure could be used to get an appropriate value for $\delta_{t}$ using all the a priori knowledge on the system. In the next subsection a modified algorithm is proposed to relax conditions (9) and (10).

\section{B. The F-CLOE-OBE (Filtered-CLOE-OBE) algorithm.}

Here the idea is to remove the condition (9) required for stability, to this end we use an adaptation filter $F(q)$. Let first define the a priori and a posteriori adaptation errors as

$$
\left\{\begin{array}{l}
\eta_{t / t-1}=\varepsilon_{t / t-1}+(F(q)-1) \varepsilon_{t / t} \\
\eta_{t / t}=F(q) \varepsilon_{t / t}
\end{array}\right.
$$

$F(q)$ is a monic adaptation filter designed by the user. These definitions allow us to propose a filtered parameter adaptation algorithm by simply substituting in (6) and (7):

- $\varepsilon_{t / t-1}$ by $\eta_{t / t-1}$ and $\varepsilon_{t / t}$ by $\eta_{t / t}$

- $y_{t}$ by $y_{t}^{F}$ such that $F(q) y_{t}^{F}=y_{t}$ and $\hat{\phi}_{t}$ by $\widehat{\phi}^{F}{ }_{t}$ such that $F(q) \widehat{\phi^{F}}{ }_{t}=\hat{\phi}_{t}$. 
The idea is to compensate the effect of $\frac{S(q)}{A(q) S(q)+q^{-d} B(q) R(q)}$ in (9) and (10).

Taking into account these adjustments, the two following equations sets summarize the proposed Filtered CLOE-OBE algorithm:

$$
\left\{\begin{array}{l}
\hat{\theta}_{t}=\hat{\theta}_{t-1}+\Gamma_{t} \eta_{t / t-1} \\
\Gamma_{t}=\frac{P_{t-1} \widehat{\phi}^{F}{ }_{t} \sigma_{t}}{\lambda+\widehat{\phi}^{F}{ }_{t} P_{t-1} \widehat{\phi}^{F}{ }_{t} \sigma_{t}} \\
P_{t}=\frac{1}{\lambda}\left(I_{n}-\Gamma_{t} \widehat{\phi}^{F}{ }_{t}^{T}\right) P_{t-1} \\
\varepsilon_{t / t-1}=y_{t}^{F}-\widehat{\phi}^{F}{ }_{t}^{T} \hat{\theta}_{t-1}
\end{array}\right.
$$

with

$$
\sigma_{t}=\left\{\begin{array}{l}
\frac{\lambda}{{\widehat{\phi^{F}}}_{t}^{T} P_{t-1} \widehat{\phi}_{t}}\left(\left|\frac{\eta_{t / t-1}}{\delta_{t}}\right|-1\right) \\
\quad \text { if }\left(\left|\eta_{t / t-1}\right|>\delta_{t}\right) \text { and }\left(\widehat{\phi}^{F}{ }_{t}^{T} P_{t-1}{\widehat{\phi^{F}}}_{t}>0\right) \\
0 \text { otherwise }
\end{array}\right.
$$

It can easily be established that if the persistent excitation condition is satisfied then the following key property holds:

$$
\forall \sigma_{t} \quad\left|\eta_{t / t}\right| \leq \delta_{t}
$$

bearing in mind that $\delta_{t}$ is a now a bound on the a posteriori adaptation error $\eta_{t / t}$.

Here the equation of the a posteriori prediction error is:

$$
\varepsilon_{t / t}=\frac{S(q)}{A(q) S(q)+q^{-d} B(q) R(q)}{\widehat{\phi^{F}}}_{t}^{T} \tilde{\theta}_{t}+\frac{1}{F(q)} v_{t}
$$

which gives

$$
\eta_{t / t}=F(q) \frac{S(q)}{A(q) S(q)+q^{-d} B(q) R(q)}{\widehat{\phi^{F}}}_{t}^{T} \tilde{\theta}_{t}+v_{t}
$$

Using this last equation, the following result presents an analysis of the proposed F-CLOE-OBE algorithm.

Result 2: Consider the class of systems defined in section II and the F-CLOE-OBE algorithm given by (15) and (16). Assume that $F(q)$ and $G(q)$ are such that

$$
\left\|1-\frac{A(q) S(q)+q^{-d} B(q) R(q)}{S(q)} \frac{1}{F(q)}\right\|_{1}<1
$$

- $\delta_{t}$ is such that:

$$
\delta_{t} \geq \frac{\left\|\frac{A(q) S(q)+q^{-d} B(q) R(q)}{S(q)} \frac{1}{F(q)}\right\|_{1}}{1-\left\|1-\frac{A(q) S(q)+q^{-d} B(q) R(q)}{S(q)} \frac{1}{F(q)}\right\|_{1}} \delta_{v_{t}}
$$

then for all initial conditions

$$
\left|\tilde{\theta}_{t}\right|^{2} \leq \gamma_{1}\left|\tilde{\theta}_{0}\right|^{2}
$$

If, furthermore, $\left\{\widehat{\phi}_{t}{ }_{t}\right\}$ is a persistently exciting sequence of order $o_{e} \geq n$, then the following properties hold:

- for all $t \geq o_{e}+1$

$$
\left|\tilde{\theta}_{t}\right|^{2} \leq \gamma_{2} \lambda^{t}\left|\tilde{\theta}_{0}\right|^{2}
$$

- For $\lambda<1$ one has

$$
\lim _{t \rightarrow \infty}\left|\eta_{t / t-1}\right| \leq \delta_{t}
$$

Proof: The proof of this result follows the same scheme that the proof of result 1 , the difference lies in the relation between $\eta_{t / t}$ and $\tilde{\theta}_{t}$.

The ideal filter is $F(q)=\frac{A(q) S(q)+q^{-d} B(q) R(q)}{S(q)}$. Thus, conditions (17) and (18) are much milder than conditions (9) and (10) if a reasonable estimated model is available. This remark leads to the following iterative identification scheme:

1) Choose a high threshold $\delta_{t}$ and apply the CLOE-OBE algorithm to get $\widehat{G}(q)$;

2) Given this first estimation, design a filter

$$
F(q)=\frac{\widehat{A}(q) S(q)+q^{-d} \widehat{B}(q) R(q)}{S(q)}
$$

and choose a lower $\delta_{t}$;

3) Apply the F-CLOE-OBE algorithm to get a new $\widehat{G}(q)$;

4) Repeat steps 2 and 3 until convergence of step 3.

The first step is an initialization step: an initial estimate of the model is necessary so as to implement this F-CLOEOBE algorithm. It is difficult to make a general discussion on the behavior of that iterative scheme and no global convergence results are available (not more than for the recursive maximum likelihood algorithm [13]), however it is successfully applied on a numerical example in the next section.

Note that if $F(q) \simeq \frac{A(q) S(q)+q^{-d} B(q) R(q)}{S(q)}$ it is possible to choose $\delta_{t}=\delta_{v_{t}}$. In that case, from (21) we have $\lim _{t \rightarrow \infty} \hat{\theta}_{t}=$ $\hat{\theta}$ where $\hat{\theta}$ is such that

$$
\left|\eta_{t}\right| \leq \delta_{v_{t}}
$$

with $\eta_{t}=F(q) \varepsilon_{t}=F(q)\left(y_{t}^{F}-{\widehat{\phi^{F}}}_{t}^{T} \hat{\theta}\right)=y_{t}-\widehat{\phi}_{t}^{T} \hat{\theta}$. Then the contribution of the filter $F(q)$ is twofold: it relaxes stability condition of the algorithm and it allows the estimation of a model $\widehat{G}(q)$ such that:

$$
\left|\left(y_{t}-\frac{\widehat{G}(q)}{1+\widehat{G}(q) C(q)} r_{t}\right)\right| \leq \delta_{v_{t}}
$$

which is coherent with (2), (3) and (4).

\section{SIMULATION RESULTS}

Numerical data have been generated according to (2), (3) and (4). The system and the controller are the following:

$$
G_{o}(q)=\frac{q^{-1}+0.1 q^{-2}}{1-1.8 q^{-1}+0.7 q^{-2}} \quad C(q)=\frac{0.1}{1-0.4 q^{-1}}
$$


TABLE I

NUMERICAL VALUES OF THE TRUE PARAMETERS AND THE ESTIMATED PARAMETERS

\begin{tabular}{|c|c|c|c|c|}
\hline parameter & $\boldsymbol{\theta}^{(1)}$ & $\boldsymbol{\theta}^{(2)}$ & $\boldsymbol{\theta}^{(3)}$ & $\boldsymbol{\theta}^{(4)}$ \\
\hline \hline real value & $b_{0}=1$ & $b_{1}=0.1$ & $a_{1}=-1.8$ & $a_{2}=0.7$ \\
\hline Procedure 1 & 0.6498 & 1.2544 & -1.4893 & 0.2952 \\
\hline Procedure 2 & 0.7412 & 0.4164 & -1.7398 & 0.6351 \\
\hline
\end{tabular}

Let notice that the plant is unstable and condition (9) in result 1 is violated in this example.

The exogenous input $r_{t}$ is a random binary sequence of length $N=4000 . v_{t}$ is a noise generated in the following manner:

$$
v_{t}=\delta_{v_{t}} \frac{1}{2}\left(e_{t}+\sin (\pi t / 10)\right)
$$

where $e_{t}$ is a white noise uniformly distributed in $[-1 ; 1]$ and $\delta_{v_{t}}=5$ (this corresponds to a signal to noise ratio of $9.12 \mathrm{~dB}$ ). The first half of the data has been used in the estimation step, the second half has been used in the validation step.

It can easily be shown that condition (9) in result 1 is not satisfied in this example. However two identification procedures have been used:

- Procedure 1: even if condition (9) is not satisfied, we can try to use the CLOE-OBE algorithm to identify the system. This is the first identification procedure.

- Procedure 2: this second identification procedure is our iterative scheme. This scheme uses the F-CLOE-OBE algorithm and has been applied over 10 iterations (on this example the time required to obtain the convergence of the estimates).

For each procedure we have chosen the threshold $\delta_{t}$ as follows:

- Procedure 1: $\delta_{t}=\delta_{v_{t}}$.

- Procedure 2: $\delta_{t}$ : we have chosen a decreasing threshold. At iteration $i$ we have

$$
\delta_{t}^{(i)}=\left(\delta_{t}^{(i n i)}-\delta_{t}^{(f i n)}\right) e^{-i}+\delta_{t}^{(f i n)}
$$

with $\delta_{t}^{(i n i)}=5 \delta_{v_{t}}$ and $\delta_{t}^{(f i n)}=\delta_{v_{t}}$.

The following results correspond to the estimates made on a single data series.

Fig. 3 presents the improvement of the estimated vector at the end of each iteration in the iterative scheme. It appears that the iterative procedure allows a clear convergence of the estimated parameters to their true value. This is confirmed by Fig. 4 which gives the ideal filter $F(q)$ and its successive estimates. Fig. 5 shows the convergence of the estimated models towards the nominal system. Note that on other applications, it is possible that the number of iterations required for convergence is different from 10 .

The table I confirms the good performance of the proposed iterative scheme compared to the algorithm CLOE-OBE: the
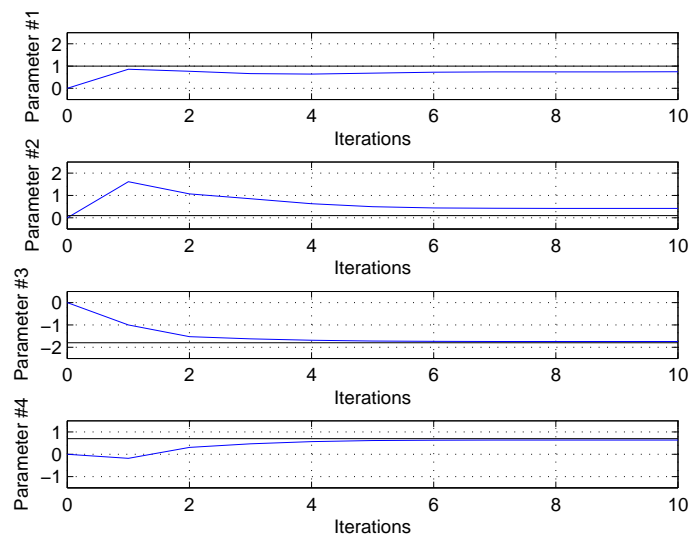

Fig. 3. Convergence of parameters

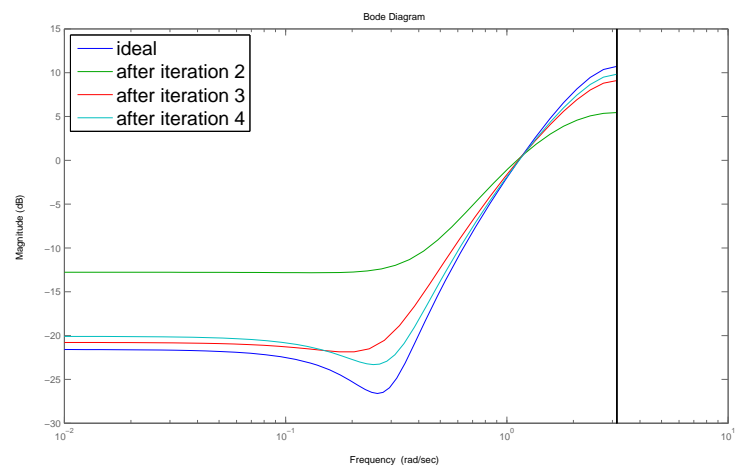

Fig. 4. Bode diagrams of ideal filter $F(q)=\frac{A(q) S(q)+q^{-d} B(q) R(q)}{S(q)}$ and its estimates

use of the filter $F(q)$ in the algorithm improve the quality of the estimated parameters. The closeness of the estimated parameters to real parameters depends on the noise level $\delta_{v_{t}}$. Fig. 6 allows the comparison between $\frac{G_{o}(q)}{1+G_{o}(q) C(q)}$ and its estimates $\frac{\widehat{G}(q)}{1+\widehat{G}(q) C(q)}$ with procedure 1 and procedure 2 . One can conclude from these Bode diagrams that the developed iterative method works well.

This is confirmed by Fig. 7 which presents thresholds $\pm \delta_{v_{t}}$ and the output errors $y_{t}-\frac{\widehat{G}(q)}{1+\widehat{G}(q) C(q)} r_{t}$ for each estimated model (model for procedure 1 and final model for procedure 2 at the $10^{\text {th }}$ iteration). It appears that the model obtained with the iterative scheme using the F-CLOE-OBE is the only one satisfying (22).

\section{CONCLUSION}

In this study, we have proposed two algorithms allowing the identification of a system operating in closed loop and subject to bounded disturbances. The second algorithm is a filtered adaptation of the first one, the introduced filter is used to relax some sufficient conditions for stability and convergence. Using the second algorithm in an iterative scheme has lead to a significant improvement of the estima- 


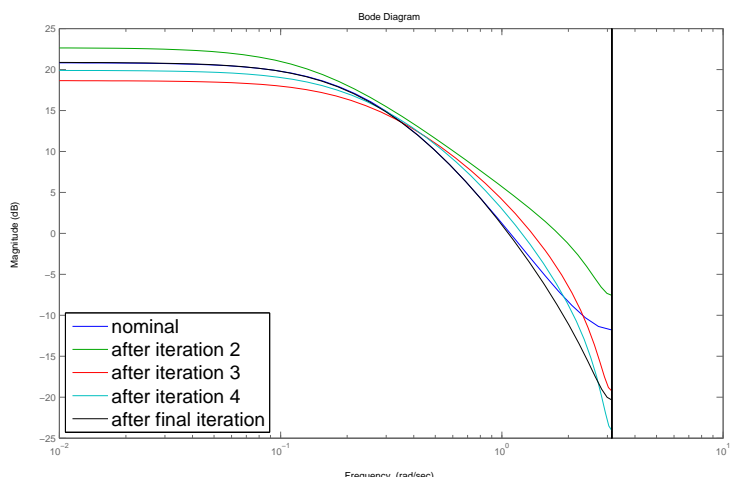

Fig. 5. Bode diagrams of the nominal system $G_{o}(q)$ and its estimates with procedure 2

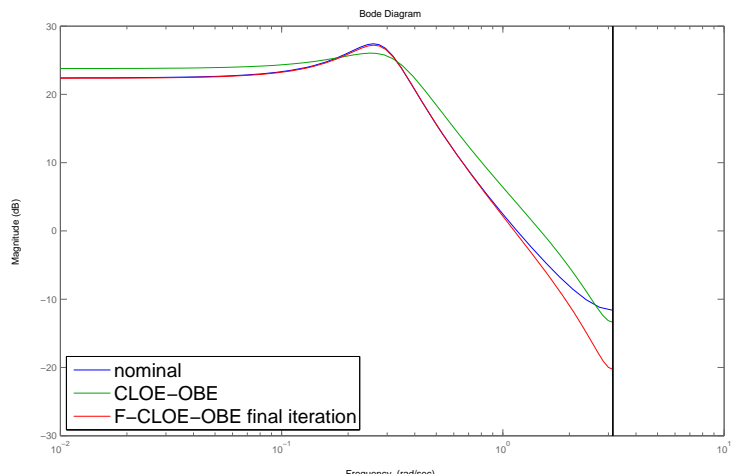

Fig. 6. Comparison between $\frac{G_{o}(q)}{1+G_{o}(q) C(q)}$ and its estimates $\frac{\widehat{G}(q)}{1+\widehat{G}(q) C(q)}$ with procedure 1 and 2

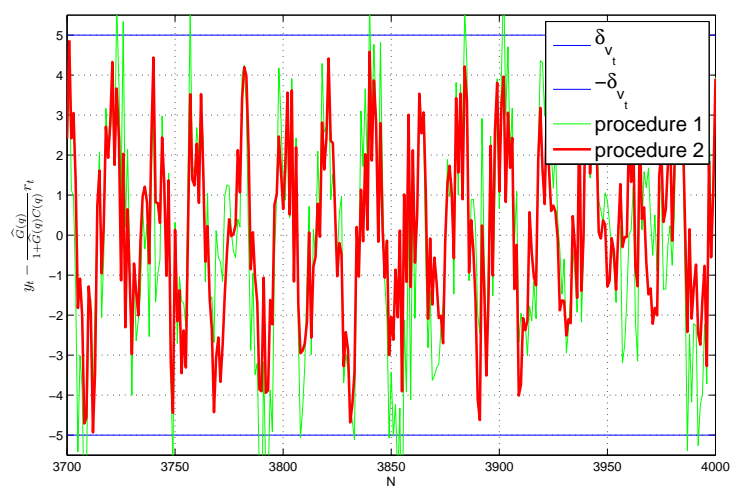

Fig. 7. Closed loop output error with estimated models tion in a numerical example. In terms of perspective, some comparisons with other methods will be presented. Moreover it might be interesting to analyze the set membership of the estimated parameters compared to the true values and it might also be interesting to propose alternative solutions to relax stability and convergence conditions.

\section{REFERENCES}

[1] C. Canudas-De-Wit and J. Carrillo. "A modified EW-RLS algorithm for systems with bounded noise". Automatica, 26 (3),599-606, 1990.

[2] M. Casini and A. Garulli and A. Vicino. "On input design in 11 conditional set membership identification". Automatica, 42 (5),815823, 2006.

[3] B. Codrons and B.D.O. Anderson and M. Gevers. "Closed loop identification with an unstable or nonminimum phase controller". Automatica, 38 (12),2127-2137, 2002.

[4] S. Dasgupta and Y.F. Huang. "Asymptotically convergent modified recursive least square with data-dependent updating and forgetting factor for systems with bounded noise". IEEE Transactions on information theory, 33 (3),383-392, 1987.

[5] E. Fogel and Y.F. Huang. "On the value of informaton in system identification - bounded noise case". Automatica, 18 (2),229-238, 1982.

[6] U. Forssell and L. Ljung. "Closed-loop identification revisited". Automatica, 35, 1215-1241, 1999.

[7] A. Garulli and B.Z. Kacewicz and A. Vicino and G. Zappa. 'Error bounds for conditional algorithms in restricted complexity set membership identification". IEEE Transactions on automatic control, 45, 160164, 2000.

[8] A. Garulli, A. Vicino and G. Zappa. "Conditional central algorithms for worst-case set membership indentification and filtering". IEEE Transactions on automatic control, 45, 1423, 2000.

[9] M. Gilson and P.M.J. Van Den Hof. "Instrumental variable methods for closed loop system identification". Automatica, 41 (2),241-249, 2005.

[10] I.D. Landau and A. Karimi. "An output error recursive algorithm for unbiased identification in closed loop". Automatica, 33(5), 933-938, 1997.

[11] I.D. Landau and A. Karimi. "Recursive algorithms for identification in closed loop: a unified approach and evaluation”. Automatica, 33(8), 1499-1523, 1997.

[12] I.D. Landau and R. Lozano and M. MSaad "Adaptive control". Springer, 1998.

[13] L. Ljung. "System identification: theory for the user". Prentice Hall, 1999.

[14] R. Lozano-Leal and R. Ortega. "Reformulation of the parameter identification problem for systems with bounded disturbances". Automatica, 23 (2),247-251, 1987.

[15] M. Milanese and G. Belforte. "Estimation theory and uncertainty intervals evaluation in presence of unknown but bounded errors; linear families of models and estimators". IEEE Transactions on automatic control, 27,408-414, 1982.

[16] M. Pouliquen and E. Pigeon and O. Gehan. "Output error identification for multi-input multi-output systems with bounded disturbances". Control and Decision Conference - European Control Conference, Orlando, 2011.

[17] A.K. Rao and Y.F. Huang. "Recent developments in optimal bounding ellipsoidal parameter estimation". Mathematics and Computers in simulation, 32, 1990.

[18] T. Söderström and P. Stoica. "System identification". Prentice Hall, 1989.

[19] G. Tan and C. Wen and Y.C. Soh. "Identification for systems with bounded noise". IEEE Transactions on automatic control, 42 (7), 9981001, 1997.

[20] P.M.J. Van Den Hof and R.J.P. Schrama. "An indirect method for transfer function estimation from closed loop data". Automatica, 29, 1523-1527, 1993.

[21] A. Vicino and G. Zappa. "Sequential approximation of parameter sets for identification with parametric and nonparametric uncertainty". Control and Decision Conference, New York, 1993. 